\title{
Editorial
}

\section{Epidemiologia e Serviços de Saúde, 2011 a 2021: uma década virtuosa \\ doi: 10.1590/S1679-49742021000300001}

\section{Epidemiology and Health Services, 2011-2021: a virtuous decade}

\section{Epidemiología y Servicios de Salud, 2011 a 2021: una década virtuosa}

\begin{abstract}
A Epidemiologia e Serviços de Saúde: revista do Sistema Único de Saúde do Brasil (RESS) foi criada em 1992, como Informe Epidemiológico do SUS (IESUS), e, em 2003, adotou o formato de revista científica. ${ }^{1}$ Em 2011, a partir das sólidas bases construídas em seu histórico, iniciou-se uma nova fase de impulso ao desenvolvimento da revista, com a ampliação do Comitê Editorial e da equipe editorial. ${ }^{2,3}$

Procedeu-se à revisão do plano de fortalecimento da RESS, visando reforçar o caráter científico do periódico e alcançar padrões de publicação para atendimento aos critérios de indexação de bases bibliográficas nacionais e internacionais. As instruções aos autores foram revisadas, a submissão de manuscritos via correio foi abandonada e o processo editorial passou a ser realizado de forma totalmente eletrônica. Foram realizados esforços para assegurar a publicação de 15 artigos originais por número, com regularidade e pontualidade. Como resultado, em um curto intervalo de tempo, a RESS foi indexada nas coleções SciELO Brasil (2014) ${ }^{4}$ e SciELO Saúde Pública (2015), assim como nas bases bibliográficas Medline (2016), ${ }^{5}$ Scopus (2017), Embase (2017), Emerging Sources Citation Index (2017) ${ }^{6}$ e AmeliCA (2020).
\end{abstract}

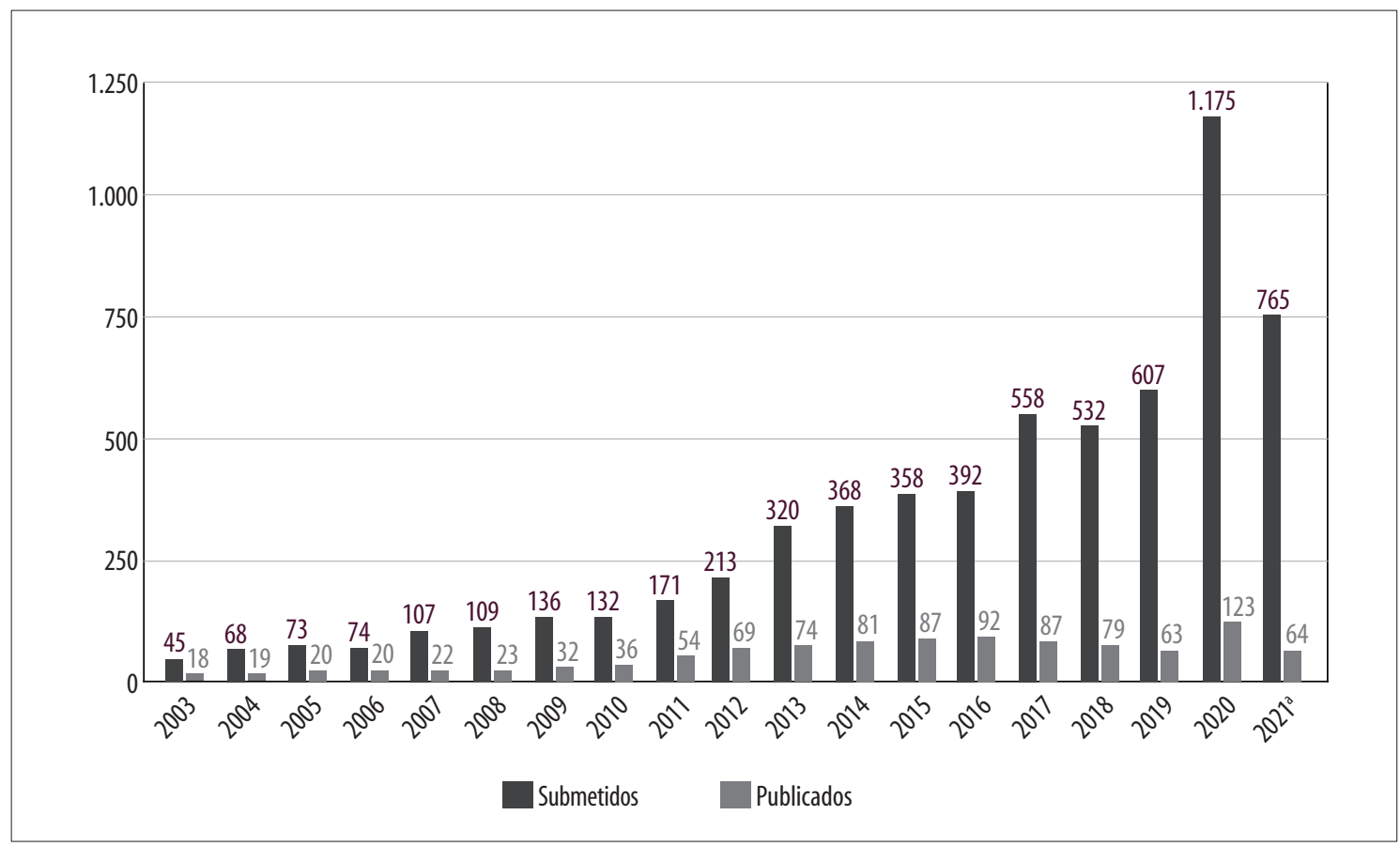

a) Número de artigos publicados até o número 3 de 2021; número de artigos submetidos até31 de julho de 2021.

Figura 1 - Número de artigos submetidos e publicados na Epidemiologia e Serviços de Saúde: revista do Sistema Único de Saúde do Brasil, 2003-2021 
A indexação na Coleção SciELO Brasil foi fundamental para impulsar a profissionalização do processo editorial da RESS. A revista passou a utilizar sistema eletrônico de gestão de manuscritos e começou a publicar todos os artigos nas versões em português e inglês, com resumos em versões nesses idiomas, bem como em espanhol. ${ }^{3} 0$ período de 2011 a 2021 foi caracterizado pela ampliação da relevância da RESS na área da Saúde Coletiva, e sua consolidação como periódico científico com qualidade e reconhecimento nacional e internacional.,

Nessa década virtuosa, a revista apresentou expressivo crescimento no número de artigos submetidos e publicados (Figura 1), bem como incremento de seus indicadores de acesso e citação, com consequente melhoria na classificação Qualis CAPES. ${ }^{8}$ Do número 4 de 2011 ao número 3 de 2021, foram publicados 39 números regulares, incluindo 641 artigos originais ou de revisão. Tais artigos, assim como os editoriais, abrangeram temáticas diversas, acompanhando a evolução da epidemiologia e da vigilância em saúde no período e destacando temas relevantes, a exemplo da epidemia de microcefalia pelo vírus Zika no Brasil (2015-2016) ${ }^{9-11}$ e da pandemia da COVID-19 (de 2020 até 0 momento). ${ }^{12-17}$ Também foram publicados artigos de séries temáticas, orientações para a redação científica, versões em português de documentos internacionais contendo recomendações para a publicação e orientações sobre integridade científica, e guias de redação científica. ${ }^{3}$ Ademais, foram publicados três números especiais que destacaram assuntos relevantes na área da vigilância em saúde. ${ }^{18-20}$

0 período também foi marcado pela realização de atividades de divulgação científica, de formação de autores e revisores, e pelo estabelecimento e fortalecimento de parcerias com outras revistas da área, a exemplo da Revista Brasileira de Epidemiologia, da Revista da Sociedade Brasileira de Medicina Tropical e da Revista Pan-Amazônica de Saúde. Destaca-se a criação, em 2012, do Prêmio RESS Evidencia, para reconhecimento ao melhor artigo original publicado na RESS a cada ano. ${ }^{3}$

Participar da evolução da RESS, durante esta década virtuosa - de 2011 a 2013, como Editora Científica, e de 2014 a 2021, como Editora Geral -, foi uma grande satisfação e um privilégio. Os resultados alcançados, fruto de intenso trabalho e esforços coletivos, devem ser celebrados. Agradeço a toda a equipe da revista, aos membros do Comitê Editorial, gestoras e gestores da Secretaria de Vigilância em Saúde (SVS/MS), autoras, autores e a toda a comunidade da RESS, que colaboraram para que a revista lograsse grande desenvolvimento. Menciono especialmente $o$ Dr. Jarbas Barbosa, que, com sua extraordinária capacidade de liderança e entusiasmo, sempre apoiou o desenvolvimento da RESS, desde seu princípio. Também, a Profa. Dra. Elisete Duarte, que atuou com extrema competência, dedicação e integridade como Editora Executiva da RESS, ao longo dessa década.

A transição na gestão da RESS ocorreu em um momento no qual o país apresentava expressivos números de casos e mortes por COVID-19, com desastrosas consequências sociais e econômicas, enquanto gestores públicos com posições anticientíficas estavam responsáveis pelo enfrentamento à epidemia. Nessa situação adversa, cumpre destacar o papel fundamental do Comitê Editorial da RESS, incansável no exercício de suas atribuições e no zelo pela autonomia da revista perante sua instituição mantenedora. Membros do Comitê se posicionaram de forma enfática para garantir que a indicação da gestão da SVS para a Editoria Geral fosse realizada em conformidade com o Estatuto da revista.

Com orgulho, e aproveitando a rica experiência acumulada, passo a compor este honrado Comitê, que seguirá atuando para promover o constante aprimoramento da RESS e para que a revista continue a cumprir sua nobre missão de difundir o conhecimento epidemiológico aplicável às ações de vigilância, de prevenção e de controle de doenças e agravos de interesse da Saúde Pública, visando ao aprimoramento dos serviços oferecidos pelo SUS.

Vida longa à RESS!

Leila Posenato Garcia' - (o orcid.org/0000-0003-1146-2641

1'Fundação Jorge Duprat Figueiredo de Segurança e Medicina do Trabalho, Centro Regional Sul, Florianópolis, SC, Brasil

\section{Referências}

1. Garcia LP, Duarte E. Epidemiologia e serviços de saúde: a trajetória da revista do Sistema Único de Saúde do Brasil. Cienc Saude Colet. 2015;20(7):2081-90. doi: https://doi.org/10.1590/1413-81232015207.06122015. 
2. Garcia LP, Duarte E. Fortalecendo a revista do Sistema Único de Saúde. Epidemiol Serv Saude. 2011;20(3):273. doi: http:// dx.doi.org/10.5123/S1679-49742011000300001.

3. Reis JG, Duarte E, Eble LJ, Garcia LP. Epidemiologia e serviços de saúde: 25 anos em revista. Epidemiol Serv Saude. 2017;26(4):685-700. doi: http://dx.doi.org/10.5123/s1679-49742017000400002.

4. Garcia LP, Duarte E. A revista Epidemiologia e Serviços de Saúde ingressa na coleção SciELO Brasil. Epidemiol Serv Saude. 2014;23(3):387-8. doi: https://doi.org/10.5123/S1679-49742014000300001.

5. Cavalcante AL, Garcia LP. Os 25 anos da Epidemiologia e Serviços de Saúde: revista do Sistema Único de Saúde do Brasil e sua indexação na base MEDLINE®. Epidemiol Serv Saude. 2017;26(1):5-6. doi: https://doi.org/10.5123/S167949742017000100001.

6. Garcia LP, Duarte E. Epidemiologia e Serviços de Saúde: história, conquistas e futuro. Epidemiol Serv Saude. 2017;26(4):683-4. doi: https://doi.org/10.5123/\$1679-49742017000400001.

7. Duarte E, Garcia LP. Revisitando a trajetória da Epidemiologia e Serviços de Saúde: o ciclo virtuoso de 2011 a 2018. Epidemiol Serv Saude. 2018;27(4):e2018418. doi: https://doi.org/10.5123/S1679-49742018000400018.

8. Garcia LP, Duarte E. Epidemiologia e Serviços de Saúde: revista do Sistema Único de Saúde do Brasil classificada como Qualis/Capes B2 na área da Saúde Coletiva. Epidemiol Serv Saude. 2017;26(2):243-4. doi: https://doi.org/10.5123/S167949742017000200001.

9. Henriques CMP, Duarte E, Garcia LP. Desafios para o enfrentamento da epidemia de microcefalia. Epidemiol Serv Saude. 2016;25(1):7-10. doi: https://doi.org/10.5123/\$1679-49742016000100001.

10. Duarte E, Garcia LP. Pesquisa e desenvolvimento para o enfrentamento da epidemia pelo vírus Zika e suas complicações. Epidemiol Serv Saude. 2016;25(2):231-2. doi: https://doi.org/10.5123/S1679-49742016000200001.

11. Garcia LP, Duarte E. Evidências da vigilância epidemiológica para o avanço do conhecimento sobre a epidemia do vírus Zika. Epidemiol Serv Saude. 2016;25(4):679-81. doi: https://doi.org/10.5123/S1679-49742016000400001..

12. Croda JHR, Garcia LP. Resposta imediata da Vigilância em Saúde à epidemia da COVID-19. Epidemiol Serv Saude. 2020;29(1):e2020002. doi: https://doi.org/10.5123/S1679-49742020000100021.

13. Garcia LP, Duarte E. Intervenções não farmacológicas para o enfrentamento à epidemia da COVID-19 no Brasil. Epidemiol Serv Saude. 2020;29(2):e2020222. doi: https://doi.org/10.5123/\$1679-49742020000200009.

14. Oliveira WK, Duarte E, França GVA, Garcia LP. Como o Brasil pode deter a covid-19. Epidemiol Serv Saude. 2020;29(2):e2020044. doi: https://doi.org/10.5123/\$1679-49742020000200023.

15. Garcia, LP. Dimensões de sexo, gênero e raça na pesquisa sobre covid-19. Epidemiol Serv Saude: 2020;29(3):e20202207. doi: https://doi.org/10.5123/S1679-49742020000300023.

16. Garcia LP, Duarte E. Infodemia: excesso de quantidade em detrimento da qualidade das informações sobre a covid-19. Epidemiol Serv Saude. 2020;29(4):e2020186. doi: https://doi.org/10.1590/S1679-49742020000400019.

17. Garcia, Leila Posenato. Gratidão ao Sistema Único de Saúde do Brasil. Epidemiologia e Serviços de Saúde [online]. 2020, v. 29, n. 5 [Acessado 11 Agosto 2021] , e2020333. Disponível em: <https://doi.org/10.1590/s167949742020000500024>. Epub 16 Dez 2020. ISSN 2237-9622. https://doi.org/10.1590/S1679-49742020000500024.

18. Garcia LP Duarte E. A contribuição do consenso brasileiro em doença de Chagas no contexto epidemiológico nacional. Epidemiol Serv Saude. 2016;25(n. spe.) :5-6. doi: https://doi.org/10.5123/S1679-49742016000500001.

19. Millington MA, Nishioka AS, Martins ST, Santos ZMG, Lima Júnior FEF, Alves RV. Paracoccidioidomicose: abordagem histórica e perspectivas de implantação da vigilância e controle. Epidemiol Serv Saude. 2018;27(n. spe.):e0500002. doi: https://doi.org/10.5123/\$1679-49742018000500002.

20. Galvão TF, Costa CHN, Garcia LP. Atenção integral às pessoas com infecções sexualmente transmissíveis. Epidemiol Serv Saude. 2021;30(n. spe.):e2020954. doi: https://doi.org/10.1590/\$1679-4974202100001.especial. 